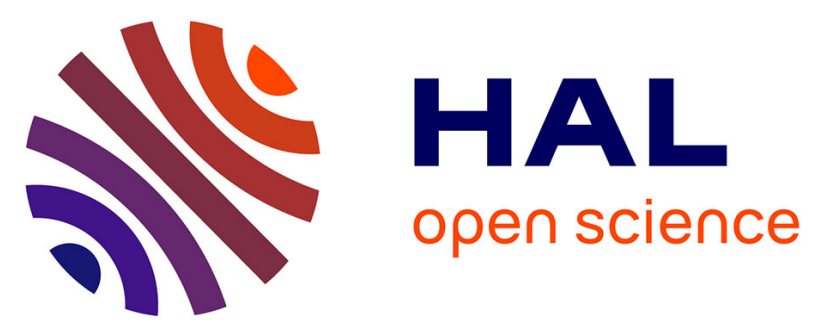

\title{
Angular scattering in fast ionatom electron transfer collisions: projectile wave diffraction and Thomas mechanisms
}

\author{
M Gudmundsson, D Fischer, N Haag, H a B Johansson, D Misra, P Reinhed, \\ H Schmidt-Böcking, R Schuch, M Schöffler, K Støchkel, et al.
}

\section{To cite this version:}

M Gudmundsson, D Fischer, N Haag, H a B Johansson, D Misra, et al.. Angular scattering in fast ionatom electron transfer collisions: projectile wave diffraction and Thomas mechanisms. Journal of Physics B: Atomic, Molecular and Optical Physics, 2010, 43 (18), pp.185209. 10.1088/09534075/43/18/185209. hal-00597867

\section{HAL Id: hal-00597867 https://hal.science/hal-00597867}

Submitted on 2 Jun 2011

HAL is a multi-disciplinary open access archive for the deposit and dissemination of scientific research documents, whether they are published or not. The documents may come from teaching and research institutions in France or abroad, or from public or private research centers.
L'archive ouverte pluridisciplinaire $\mathbf{H A L}$, est destinée au dépôt et à la diffusion de documents scientifiques de niveau recherche, publiés ou non, émanant des établissements d'enseignement et de recherche français ou étrangers, des laboratoires publics ou privés. 


\title{
Angular scattering in fast ion-atom electron-transfer collisions: Projectile wave diffraction and Thomas mechanisms
}

\author{
M. Gudmundsson, D. Fischer, N. Haag, H. A. B. Johansson, D. Misra, P. Reinhed, H. \\ Schmidt-Böcking $^{\dagger}$, R. Schuch, M. Schöffler ${ }^{\dagger}$, K. Støchkel ${ }^{\ddagger}$, H. T. Schmidt, and H. Cederquist \\ Department of Physics, Stockholm University, AlbaNova University Center, S-10691 Stockholm, Sweden \\ ${ }^{\dagger}$ Institut für Kernphysik, Goethe Universität, 60438 Frankfurt am Main, Germany \\ ${ }^{\ddagger}$ Department of Physics and Astronomy, University of Aarhus, Ny Munkegade, DK-8000 Aarhus C, Denmark
}

(Dated: August 4, 2010)

\begin{abstract}
We report experimental angular differential cross sections for double-electron capture in $\mathrm{He}^{2+}+$ He collisions and single-electron capture in $\mathrm{H}^{+}+$He collisions for the 1.3-12.5 MeV kinetic energy range. In all cases, the total cross sections are dominated by forward scattering peaks in $d \sigma / d \Omega$. The shapes and widths (but not the magnitudes) of these peaks are very similar for all energies and for capture of one or two electrons corresponding also to our measured linear increases in the transverse momentum transfers with increasing projectile velocities. These observations may be ascribed to diffraction limitations which are connected to electron transfer probabilities $P(b)$ which are significant in limited regions of $\mathrm{b}$ only. For $\mathrm{H}^{+}+\mathrm{He}$ single-electron capture we observe two additional maxima in the angular differential cross sections. We conclude that while the secondary maxima at $\sim 0.5 \mathrm{mrad}$ probably have large contributions from the Thomas proton-electron-nucleus scattering mechanism, the third maxima at $\sim 0.75 \mathrm{mrad}$ are most likely mainly due to projectile de Broglie wave diffraction.
\end{abstract}

PACS numbers: $34.70 .+\mathrm{e}, 42.50 . \mathrm{Xa}$

\section{INTRODUCTION}

Electron transfer reactions in fast ion-atom and fast ion-molecule collisions have been studied since the earliest days of quantum mechanics. During the last decade, research on fast ion-atom collisions have mainly been concentrated on investigations of ionization processes. For investigations of such processes, the invention of the reaction microscope [1], in which the momentum vectors of all outgoing particles (ions and electrons) are measured with high resolution and high efficiencies, represents a major breakthrough. For pure electron capture processes the corresponding experimental task is to measure the three momentum components for the recoiling target ions with high precision and high resolution. The COLTRIMS technique (Cold Target RecoilIon-Momentum Spectroscopy), a forerunner to reaction microscopy, is ideally suited for this task [2].

In 2006, Fischer et al. [3] reported on a COLTRIMS experiment in which two separate contributions to the recoil ion momentum distributions, and to the projectile angular scattering distributions $d \sigma / d \Omega$, were fully resolved. The dominant feature in these distributions is due to projectile scattering in the forward direction which gives a peak centered at zero transversal momentum transfer $\left(p_{\perp}=0\right)$ to the recoiling target. A corresponding peak appears already in a theoretical treatment based on the first Born approximation (in which the process is described as a single interaction between the projectile and the active electron in the field of the target nucleus or as an interaction between this electron and the target nucleus in the projectile field [4]). How- ever, for more quantitative comparisons it is clear that also second Born contributions have to be included in the theoretical treatment [5]. Fischer et al. [3] ascribed the smaller secondary peak in $d \sigma / d \Omega$ (first found experimentally by Horsdal-Pedersen et al. [6]) to the so called Thomas process, which may be accounted for by both classical [7] and quantum mechanical (second Born) descriptions [8]. In the classical picture [7], the $\mathrm{H}^{+}$projectile $(\mathrm{p})$ scatters on one target electron (e) such that this electron (e) scatters on the target nucleus $(\mathrm{N})$ and becomes bound to the projectile. Then, energy and momentum conservation requires that the electron (e), when initially at rest in the target frame, is scattered at 60 degrees with respect to the projectile trajectory and this binary (p-e) collision thus gives the characteristic proton (p-e-N) Thomas projectile scattering angle of $0.47 \mathrm{mrad}$. In general, the $(\mathrm{p}-\mathrm{e}-\mathrm{N})$ Thomas angle for a projectile of mass $\mathrm{M}_{p}$ and electron of mass $\mathrm{m}_{e}$ is

$$
(\theta)_{p-e-N}=\frac{\sqrt{3}}{2} \frac{m_{e}}{M_{p}}
$$

which is independent of the projectile velocity $\mathrm{v}_{p}$ and the target mass, while

$$
\left(p_{\perp}\right)_{p-e-N}=\frac{\sqrt{3}}{2} v_{p} m_{e}
$$

$\left(m_{e}=1\right.$ in atomic units).

In Ref. [9], the separate velocity dependencies of the total pure non-radiative single-electron capture cross section and the resolved parts of these cross sections due to the secondary peaks in $d \sigma / d \Omega$ (Thomas processes [9]) were discussed for $1.3-12.5 \mathrm{MeV} \mathrm{H}^{+}+\mathrm{He}$ collisions. In 
this paper, we report on various detailed features (peak positions and widths) of the corresponding differential cross sections $d \sigma / d \Omega, d \sigma / d \theta$ and $d \sigma / d p_{\perp}$ and compare them with the same type of information from measurements on

$$
H e^{2+}+\mathrm{He} \rightarrow \mathrm{He}+\mathrm{He}^{2+}
$$

processes in which fast $\mathrm{He}^{2+}$ projectiles with kinetic energies of $6.0 \mathrm{MeV}$ capture both electrons from He target atoms essentially at rest. For these measurements we have used a He gas-jet target $[10,11]$ and a recoil ion momentum spectrometer (cf. [12-14]) mounted inside the ion storage ring CRYRING [15]. Surprisingly, in view of what is expected for Rutherford scattering, we find that the widths of the forward scattering peaks in $d \sigma / d \Omega$ are close to the same constant value in the energy range between 1.3 and $12.5 \mathrm{MeV}$ regardless of the number of active electrons (one or two) where we also have included the double electron capture data by Schuch et al. [16] at $1.6 \mathrm{MeV}$ in the comparison. The peak positions and widths of the forward peaks for the corresponding $d \sigma / d p_{\perp}$ cross sections are found to depend linearly on the longitudinal momentum transfer $p_{\|}$- but with different linear behaviours for single- and double-electron capture. Unlike in the cases of $\mathrm{H}^{+}+\mathrm{He}$ single-electron capture, it was not possible to isolate separate secondary maxima in $d \sigma / d \Omega$ for $6.0 \mathrm{MeV} \mathrm{He} \mathrm{He}^{2+}+$ He double electron capture. Here, we will discuss possible ways to interpret the measured shapes of the differential cross sections and we will then consider both Thomas capture mechanisms and diffraction of $\mathrm{H}^{+}$and $\mathrm{He}^{2+}$ projectile de Broglie waves on "apertures" corresponding to limited spatial regions for single- and double-electron capture close to the He target nuclei. As we will show below, the latter description may account for many of the presently observed features, in particular it can explain the constant angular widths of the forward scattering peaks and the appearances of a third maximum at large angles for single-electron capture. At the same time, we find that the $p_{\perp}$-positions of the secondary maxima in $d \sigma / d p_{\perp}$ depend on $v_{p}$ as suggested by Eq. 2, which was deduced for the (p-e-N) Thomas process.

\section{EXPERIMENT}

The experiment was performed at the synchrotron, cooler and heavy ion storage ring CRYRING [15] at Stockholm University. The experimental procedure used for the $\mathrm{H}^{+}+\mathrm{He}$ measurements has been reported in ref. [9] and here we give a description of the pure doubleelectron capture $\mathrm{He}^{2+}+\mathrm{He}$ measurements, focusing on some special precautions and steps that had to be taken in this particular case.

The $\mathrm{He}^{2+}$ projectile ions were produced in an Electron Cyclotron Resonance (ECR) ion source, accelerated to $300 \mathrm{keV} / \mathrm{u}$ by a radio-frequency quadrupole, and injected in CRYRING. Ions were accumulated in the ring through series of injections before they were accelerated to $6.0 \mathrm{MeV}$. Stored ion beam currents were typically 20 $\mu \mathrm{A}$ and the ion beam diameter was reduced to about $1.5 \mathrm{~mm}$ by means of electron cooling [17]. The overlap between the $\mathrm{He}^{2+}$ ion beam and the $1.5 \mathrm{~mm}$ diameter supersonically cooled He gas jet [10,11] was optimized by moving the jet horizontally and in the direction perpendicular to the ion beam while measuring the recoil ion count rate. The gas jet target itself consists of four differentially pumped jet forming stages above the ring chamber and three differentially pumped jet-dump stages below [10]. In the present experiment, the temperature of the gas-jet nozzle was set to $160 \mathrm{~K}$ with a driving pressure of 2.5 bar, which gave typical jet-target densities of $10^{8}$ atoms $/ \mathrm{mm}^{3}$ in the interaction region. The jet was collimated by means of two skimmers to limit its diameter and the initial momentum definition of the He target atoms before the collisions [10].

The recoil ion momentum spectrometer (cf. [12-14]) consists of an extraction region, a drift tube and a position sensitive microchannel plate detector with a resistive anode. The ion beam in CRYRING is very wide during the injection phase and therefore the opening for the circulating beam in the extraction stage of the spectrometer has to be large. The geometry is very open $\left(7 \mathrm{x} 5 \mathrm{~cm}^{2}\right)$ for the circulating beam and the extraction field is provided by a series of electrodes mounted in pairs above and below (and far from) the center of the fast circulating beam. The homogeneity of the extraction field in the central part of the extraction volume was achieved by means of two square shaped plates ('Deflection plates' in fig. 1) with bias voltages, mounted at the entrance and exit for the fast circulating $\mathrm{He}^{2+}$ beam. At the same time these plates shielded the insulator rods on which the extraction plates were mounted from exposure to the stored beam and by adjusting the difference between the plate biases the recoil ion beam could also be steered horizontally. We used the conventional Wiley-McLaren, or "time-focusing", spectrometer geometry in which the drift tube has twice the length of the active part of the extraction region [18]. In the drift region we also had an electrostatic lens which by switching could be used to prevent recoil ions with certain mass to charge ratios from entering the drift region. An overview of the experimental setup and the data acquisition system is shown in fig. 1 .

The fast $\mathrm{He}^{0}$ product atoms, formed in double-electron capture reactions (3), are detected by a second position sensitive microchannel plate (MCP) detector (with a resistive anode) mounted in the straight section behind the first dipole magnet after the gas-jet target and 3.2 meters downstream of the jet-beam interaction region. A fast signal from the back of the projectile MCP serves as the start for the time-of-flight measurement (after it has 


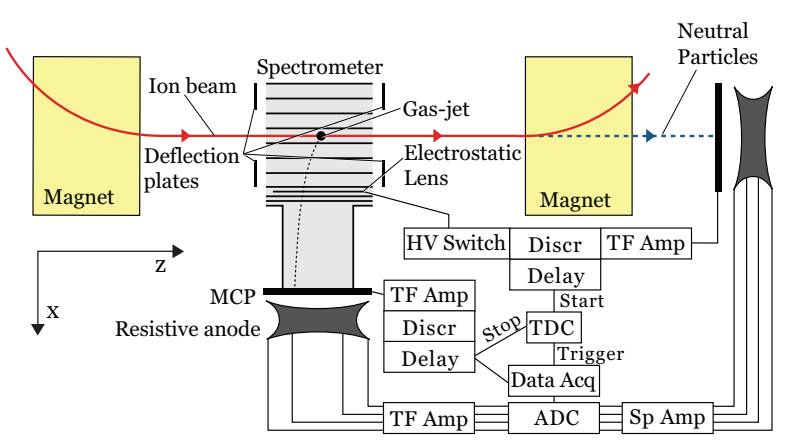

FIG. 1: Experimental setup for the $\mathrm{He}^{2+}+\mathrm{He}$ double electron capture measurements. The electrostatic lens is on a high positive voltage most of the time in order limit the random coincidence rate due to ionization processes. The lens voltage is switched to zero at a certain time after the registration of a neutral particle on the projectile detector in order to allow $\mathrm{He}^{2+}$ ions to pass and be detected first (cf. text).

been amplified, discriminated and delayed - see fig. 1). The corresponding four resistive anode corner signals are amplified and registered in list mode together with information on the time-of-flight (stop signals provided by the recoil ions) and the four recoil-detector corner signals.

At the present projectile energy of $6.0 \mathrm{MeV}$, the pure $\mathrm{He}^{2+}+\mathrm{He}$ double-electron capture cross section is in the $10^{-26} \mathrm{~cm}^{2}$ range [16]. Thus, special measures have to be taken in order to minimize random coincidence rates due to much more likely ionization processes. Such random rates are strongly reduced by means of a foil $(10 \mathrm{~mm}$ diameter) mounted centrally on a grid in front of the recoil detector. This prevents recoil ions with low momenta (such as the ones from ionization) to reach the detector, while those due to pure non-radiative capture events have substantial longitudinal momenta $p_{\|}$and do not hit the foil. This technique was used for the $\mathrm{H}^{+}+$ He measurements [9] and in the present $\mathrm{He}^{2+}+\mathrm{He}$ measurement. For the latter we did, in addition, switch the spectrometer lens voltage such that only ions within a narrow time window for the $\mathrm{He}^{2+}$ recoil ions are allowed to pass [19]. The corresponding time-of-flight spectra with and without gates on the positions on the projectile and recoil ion detectors are shown in fig. 2. Note the strong contribution from double ionization events below the double-electron capture peak in the upper spectrum without position gates, even when we use the blocking foil and switch the lens voltage on a timing gate for the $\mathrm{He}^{2+}$ recoil ions only. A further difficulty which had to be overcome was the narrow time window (about $200 \mathrm{~ns}$ ) available to record the recoil ion position (deduced from the relatively slow resistive anode signals) before the increase in count rate due to single ionization random events (cf. fig. 2, ' $\mathrm{He}^{+}$randoms'). This short time is insufficient for position detection by means of the spectroscopic amplifiers which we normally use for the resistive anode corner

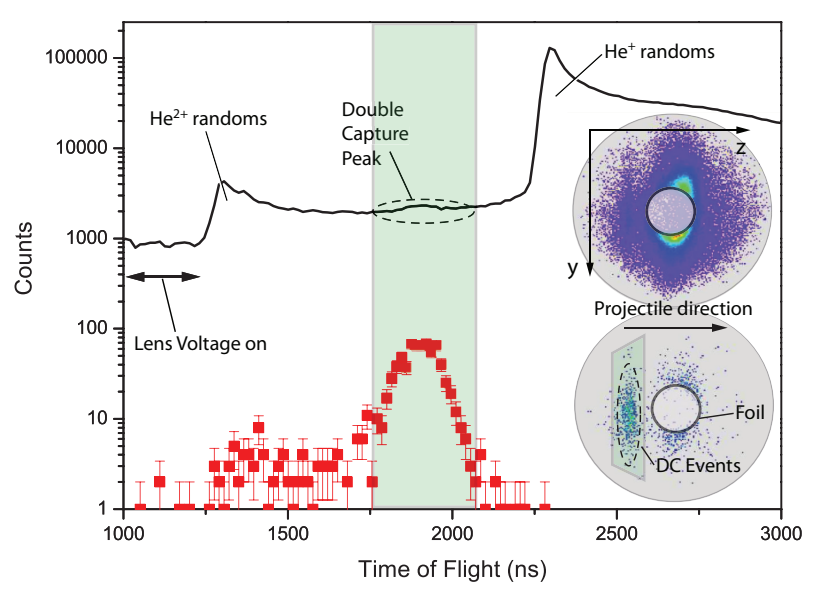

FIG. 2: Time of flight spectrum for double electron capture in 6.0 $\mathrm{MeV} \mathrm{He}^{2+}+\mathrm{He}$ collisions without position gates on the recoil and projectile detectors (upper curve) and with such gates (squares below). The shaded area shows the time of flight gate. Insets to the right show recoil detector images without (above) and with gates (below) in the time-of-flight and on the projectile detector (cf. text).

signals and, instead, much faster timing filter amplifiers were used. In the lower spectrum of fig. 2 we have used gates on the projectile detector and on the position for the double electron capture events on the recoil detector (lower inset to the right). This greatly improves the signal to noise ratio in the time of flight spectrum making the $\mathrm{He}^{2+}$ peak due to pure double-electron capture events clearly visible (cf. the lower time-of-flight spectrum in fig. 2).

\section{DATA ANALYSIS}

The momentum transfer resulting from double (or single) electron capture can be separated in one component $p_{\|}$along the direction of the fast, projectile ion beam and one component $p_{\perp}$ in the perpendicular direction. The longitudinal component along the beam can be expressed as a function of $v_{p}$, the inelasticity $Q$ and the number of transferred electrons, $n$, according to $p_{\|} \approx-n \times v_{p} / 2+Q / v_{p}$ (in atomic units). This may be approximated by $p_{\|} \approx-n \times v_{p} / 2$ as the inelasticity, given by the difference in binding energies between the initial target state and the final projectile capture state, is very small $\left(Q / v_{p}<<n v_{p} / 2\right)$. The recoil ion momentum components in the $\mathrm{y}$ - and z-directions (see the upper inset in fig. 2 where the coordinate system is defined) are obtained from the position on the detector and the time of flight whereas the momentum in the x-direction is calculated from the extraction voltage and the time of flight. The instrumental resolution in $p_{x}$ i.e. along the time-of-flight axis is about 0.3 a.u. with the present time resolution of about $3 \mathrm{~ns}$ [3], and is limited by electronics 


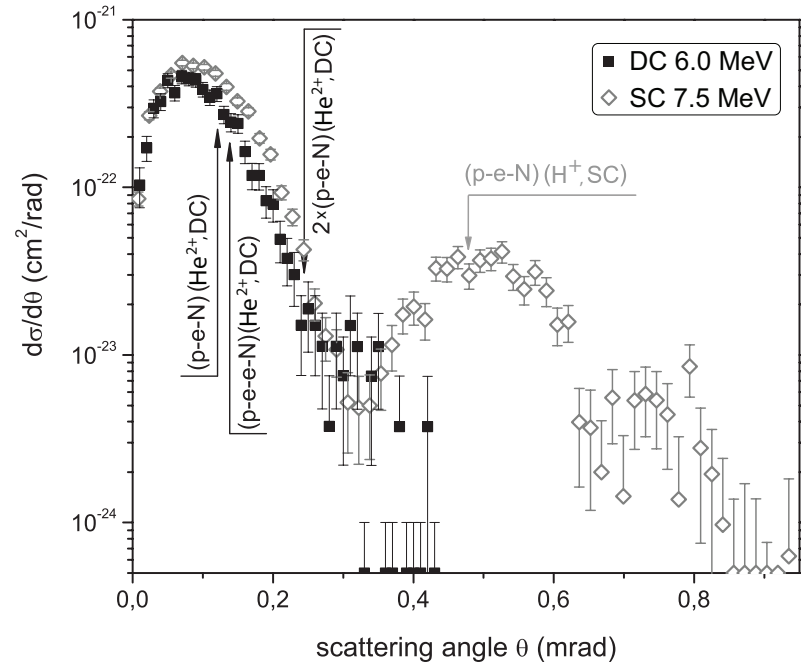

FIG. 3: Absolute angular differential cross section for $6.0 \mathrm{MeV}$ $\mathrm{He}^{2+}$ double capture (DC) from He (black boxes) and 7.5 $\mathrm{MeV}$ proton single capture (SC) from He (grey diamonds). Arrows indicate the expected angular positions for maxima of various hypothetical Thomas processes (cf. text).

and fringe field effects. The resolutions in $p_{y}$, which is determining our resulting projectile angular resolution, and $p_{z}$ depend on the image size on the detector and therefore on the extraction voltage. From the width of the double-electron capture $p_{z}$ distribution (cf. lower inset of fig. 2) we find that the $p_{z}$ (and $p_{y}$ ) resolution is about $15 \%$ of the longitudinal momentum transfer. This corresponds to scattering angle resolutions of $0.02 \mathrm{mrad}$ for $\mathrm{He}^{2+}+\mathrm{He}$ double-electron capture and $0.04 \mathrm{mrad}$ for $\mathrm{H}^{+}+\mathrm{He}$ single-electron capture. The scattering angle $\theta$ of the projectiles can in principle be measured directly on the projectile detector, or - as is the case here - it can be deduced through $\theta \approx p_{\perp} /\left(M_{p} v_{p}\right)$. The benefit of the latter method is related to the high recoil ion momentum resolution, which only depends very weakly on the size of the ion-beam/gas-jet interaction volume, and the ion beam divergence. Thus, high intensity ion beams may be used in order to reach high luminosities without degrading the resolution in the $p_{\perp}$ measurement, which then also gives high resolution in the determination of $\theta$.

\section{RESULTS AND DISCUSSION}

In figure 3 , we compare the angular differential cross sections, $d \sigma / d \theta$ for the total non-radiative $\mathrm{H}^{+}+\mathrm{He}$ single-electron capture process at $7.5 \mathrm{MeV}$ and for the total non-radiative $\mathrm{He}^{2+}+\mathrm{He}$ double-electron capture process at $6.0 \mathrm{MeV}$. In these measurements, the small contributions from radiative electron-capture processes are strongly discriminated against by means of the foil blocking the central part of the recoil-ion detector as described in the experimental section. In the single-electron

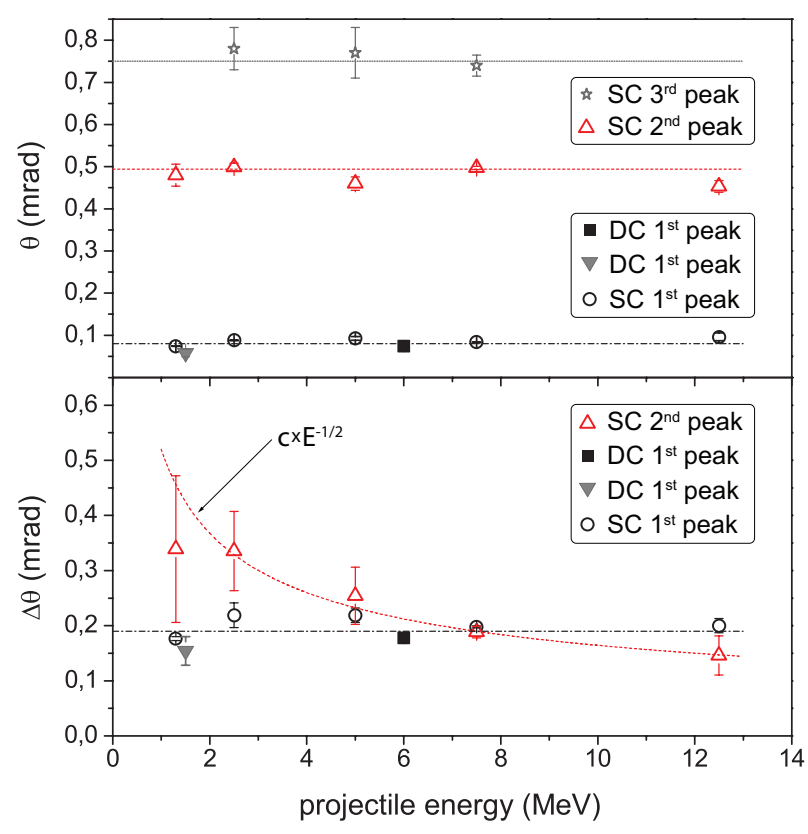

FIG. 4: Above: peak positions in $d \sigma / d \theta$ plotted as functions of the projectile energy. Below: widths (FWHM) in $\theta$ of Gaussian peaks fitted to $d \sigma / d \Omega$ plotted as functions of projectile energy.

capture data in fig. 3, additional maxima in $d \sigma / d \theta$ appear around $0.5 \mathrm{mrad}$ and $0.75 \mathrm{mrad}$. The $0.5 \mathrm{mrad} \max -$ imum is at the angle expected for the projectile-electron(target) Nucleus, or p-e-N Thomas mechanism (cf. Ref. $[3,4]$ ), while the small but significant maximum at the larger scattering angle has not been predicted theoretically.

For $\mathrm{He}^{2+}+\mathrm{He}$ double electron capture two independent single-electron Thomas transfer processes, $2 \times(\mathrm{p}-\mathrm{e}-$ $\mathrm{N}$ ) or a single $(\mathrm{p}-\mathrm{e}-\mathrm{N})$ process combined with a forward $\left(p_{\perp} \approx 0\right)$ process could in principle contribute and their expected characteristic angles are indicated by arrows in figure 3 . In addition, the angle for another doubleelectron capture process "p-e-e-N", which would also involve scattering between the He-target electrons such that both would become bound to the projectile [20] is indicated in fig. 3. Clearly, none of these three capture processes could be isolated in the present $6.0 \mathrm{MeV} \mathrm{He}^{2+}$ + He measurement.

In the upper panel of figure 4, we show characteristic scattering angles $\theta$ as functions of the collision energy defined as the the angular positions of the three maxima in $d \sigma / d \theta$ for $\mathrm{H}^{+}+\mathrm{He}$ electron capture and for the single maxima in $d \sigma / d \theta$ for $\mathrm{He}^{2+}+$ He double-electron capture at $6.0 \mathrm{MeV}$ and $1.6 \mathrm{MeV}$ (the latter double-electron capture result from Ref. [16]). All forward peaks (including those for double-electron capture) are close to a common value of $\theta=0.08 \mathrm{mrad}$. In the lower panel of figure 4 , we show the corresponding angular widths and with the exception of the secondary maxima for $\mathrm{H}^{+}+\mathrm{He}$ there 


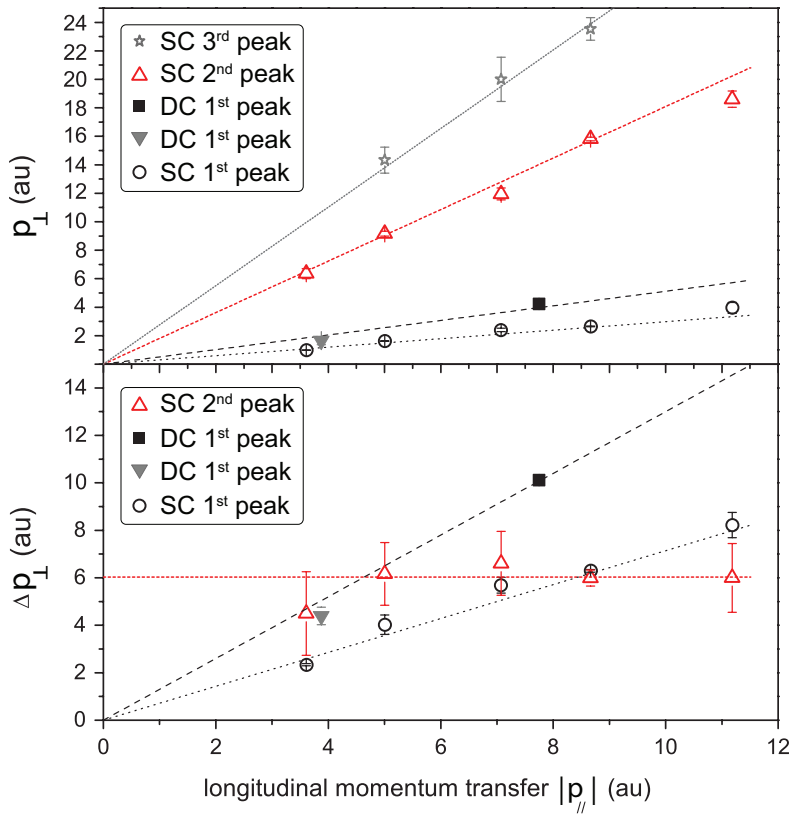

FIG. 5: Above: positions of the three maxima in $d \sigma / d p_{\perp}$ for $\mathrm{H}^{+}+$He single-electron capture and $\mathrm{He}^{2+}+\mathrm{He}$ doubleelectron capture. Below: widths (FWHM) in $p_{\perp}$ of Gaussian peaks fitted to the first two peaks of $d \sigma / d \Omega$ as a function of $\left|p_{\|}\right|$.

is very little variation with the projectile energy and as it seems - the number of transferred electrons $n$. The observed decrease in the angular widths of the secondary maxima is consistent with the results of quantum calculations of the Thomas process $[4,7]$. In the classical picture, the Thomas angle is $\theta_{p-e-N}=\sqrt{3} /\left(2 M_{p}\right)$ for an electron initially at rest in the target frame (cf. Eq. 1) and the corresponding angular width can then be expected to be given directly by the Compton profiles of the initial (target) and final (projectile capture) states. Thus, the width of the Thomas peak in $p_{\perp}$ should not change with $v_{p}$ while the corresponding $\theta$-width in $d \sigma / d \theta$ thus is expected to scale with $1 / v_{p}$ consistent with the data in fig. 4.

In figure 5, we show the measured transversal momentum transfers, $\mathrm{p}_{\perp}$ to He-target ions as functions of the corresponding longitudinal momentum transfers $p_{\|} \approx-n \times v_{p} / 2$. Note that the secondary peaks for $\mathrm{H}^{+}$ + He single-electron capture fall on a common straight line with a slope very close to $\sqrt{3}$ as expected for Thomas capture [7] as $\left(p_{\perp}\right)_{p-e-N}=\sqrt{3} p_{\|},\left(\left(p_{\perp}\right)_{p-e-N}=v_{p} \frac{\sqrt{3}}{2}\right.$, $\left.p_{\|}=v_{p} / 2\right)$. The single- and double-electron capture forward scattering peaks follow separate linear $p_{\perp}=p_{\perp}\left(p_{\|}\right)$scalings in the upper panel of fig. 5 . The $p_{\perp}$ momentum widths (fig. 5, lower panel) of the forward peaks also scale linearly with $p_{\|}$(and $v_{p}$ ) and again with different such scalings for single- and double-electron capture.

While the linear increase of $p_{\|}$with $v_{p}$ is a trivial consequence of the overall momentum and energy conser- vation, the reason why $p_{\perp}$ also in the forward capture cases $\left(1^{\text {st }}\right.$ maxima in $\left.d \sigma / d \theta\right)$ is proportional to $v_{p}$ is not immediately clear. From the description in [4] where forward capture is described as a single projectile-electron interaction in the field of the nucleus, it appears that the strength of the interaction with the nucleus would have to increase with $v_{p}$ to facilitate the larger longitudinal momentum transfers needed for electron-capture at higher velocities. However, if we now for a moment assume that the presently measured transversal momentum transfers are dominated by proton-He nuclear scattering without screening, the classical Rutherford formula would e.g. indicate an impact parameter distribution peaking at about 0.015 au to give a peak at the observed angle in $d \sigma / d \theta$ for 7.5 MeV protons. Such a strong localization of projectile trajectories in the plane perpendicular to $\bar{v}_{p}$ would however according to the uncertainty principle lead to a spread in $p_{\perp}$ of more than 30 au - much larger than the measured value of $p_{\perp}=2.65 \pm 0.03 \mathrm{au}$. Thus, we conclude that the Rutherford scattering, as predicted by Miraglia et al. [21], is not the dominating contributor to the transverse momentum transfer.

With less strict localization in the impact parameter plane, the minimum transverse momentum transfer allowed by the uncertainty principle will be smaller and may reproduce the observed distributions. In the following we deduce the electron-transfer probabilities as functions of impact parameter under the assumption that the shapes of the measured transverse momentum transfer distributions are due to diffraction of projectile de Broglie waves.

Here an analogy with classical optics is helpful. If we consider light diffracted when passing a small aperture, we expect that the width of the primary diffraction maximum is inversely proportional to the size of the aperture. In the present analogy an aperture would correspond to having an electron-transfer probability, $P(b)$, which is constant for values of $b$ smaller than a certain effective capture radius and zero for larger $b[\dagger]$. The optical analogy of a more realistic continuous electron transfer probability function $P(b)$ would be a screen, not with a hole, but with a position-dependent transmission function, $T(b)$. For the optical case, the light intensity distribution on a distant observation screen when a plane light wave is diffracted through the partially transparent screen is related to this transmission function. Specifically, the function describing the damping of the wave amplitude is related to the amplitude distribution of the outgoing wave through a Fourier transform. The same

[t] Note that in this analogy $P(b)$ corresponds to the transmission of the light since we are considering projectiles that come in charged and leave the interaction region as neutrals, whereas in the optical case we would observe the light that was merely transmitted. 
formal relation exists between the measured angular differential cross sections, $d \sigma / d \Omega$ and the electron-transfer probability, $P(b)$, and we can therefore deduce the latter directly from our data under the assumption that the shapes of the forward peaks are governed by diffraction phenomena. Conceptually, this approach is equivalent to the one which has been used to rationalize observations of projectile wave interference in electron transfer to fast projectiles, $\mathrm{H}^{+}$and $\mathrm{He}^{2+}$, from molecular hydrogen targets $[22,23]$.

Impact parameter dependent electron transfer probabilities $P(b)$ that reproduce the shapes and magnitudes of the forward peaks in the electron-transfer angular differential cross sections are calculated in this way from the cross section data in the range $\theta \leq 0.32 \mathrm{mrad}$ for singleelectron capture and $\theta \leq 0.28 \mathrm{mrad}$ for double-electron capture. In fig. 6 , we show such $P(b)$ distributions for $1.3-12.5 \mathrm{MeV} \mathrm{H}^{+}+$He single-electron capture and for 6.0 $\mathrm{MeV} \mathrm{He}{ }^{2+}+$ He double-electron capture. We first note that the values of these $P(b)$ are very small and decrease rapidly with increasing projectile velocity $v_{p}$. Further, the widths of the $P(b)$ distributions decrease with increasing $v_{p}$. Via the Heisenberg uncertainty principle this stronger localization translates to the observed increase in the transverse momentum transfer with increasing $v_{p}$.

For the double-capture case (lowest panel, fig. 6) we find that impact parameters smaller than about 0.15 au contribute most to the cross section. This range is about four times narrower than for the $\mathrm{H}^{+}+\mathrm{He} \mathrm{SC}$ at similar velocities most likely due to the fact that both electrons are transferred to the projectile.

In fig. 6, we also show $P(b)$ step functions corresponding to sharply defined 'apertures', corresponding to optical diffraction through a hole in a screen. The sizes of these apertures are chosen as to reproduce positions of the first minima in the angular differential cross sections and the constant value of $P(b)$ for $\mathrm{b}$ smaller than the aperture radii are chosen as to fit the measured cross sections. When we take the step function for the 7.5 $\mathrm{MeV} \mathrm{H}^{+}+\mathrm{He}$ case to calculate the full angular differential cross section, we get the result shown in fig. 7 , where we in addition to the central peak, also have higher order diffraction peaks. Here, the secondary diffraction peak is close to the Thomas peak and therefore a clear separation of the two contributions is not possible. However, we note that the secondary peak in $d \sigma / d \theta$ is measured to have the precisely the scaling of $p_{\perp}$ with $p_{\|}$as we expect for the p-e-N Thomas process. Further it has been demonstrated experimentally that the cross section corresponding to the secondary maximum scales as $v_{p}^{-11}$ [9] in agreement with the prediction of Thomas [7]. The third peak at $0.75 \mathrm{mrad}$ cannot be related to any multiple scattering mechanism in an obvious way and is likely to be dominated by diffraction effects. Clearly the true $P(b)$ is not a step function, but on the other hand, higher-order

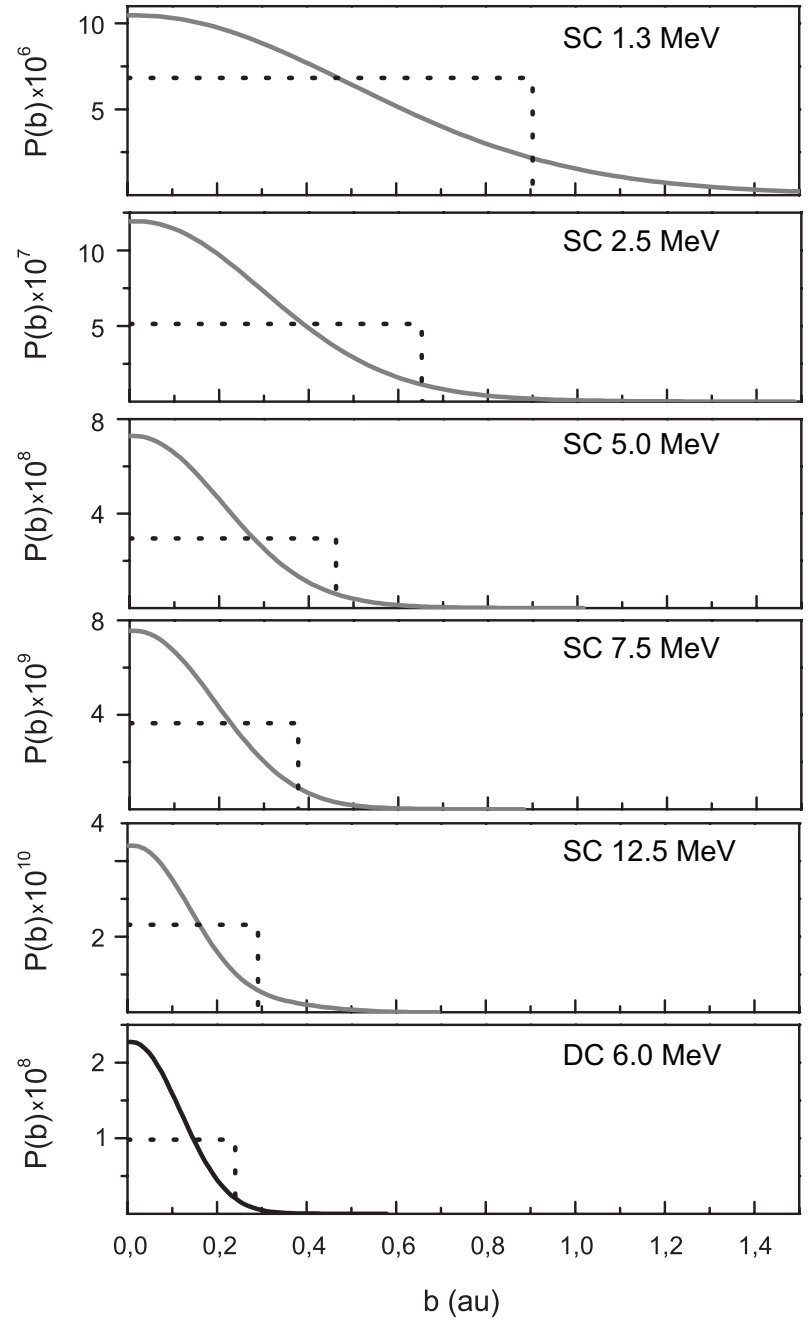

FIG. 6: Impact parameter distributions, $P(b)$, deduced from the shapes of the forward peaks in $d \sigma / d \Omega$ (cf. text) for 1.3 - $12.5 \mathrm{MeV} \mathrm{H}^{+}+$He single-electron capture (full, grey line) and 6.0 $\mathrm{MeV} \mathrm{He}^{2+}+\mathrm{He}$ double-electron capture (full, black line). Step function for $P(b)$ giving the first diffraction minima coinciding with first experimental minima at the experimental values of $0.32 \mathrm{mrad}$ and $0.28 \mathrm{mrad}$ for $\mathrm{SC}$ and DC, respectively (dotted lines).

diffraction maxima of reduced magnitude are to be expected from other non-Gaussian functional forms of $P(b)$ as well.

\section{CONCLUSION}

In this paper, we have reported that the positions and shapes of the forward (primary) scattering peaks in $d \sigma / d \theta$ are essentially independent of the collision energy and the number of transferred electrons in fast $\mathrm{H}^{+}$ $+\mathrm{He} \rightarrow \mathrm{H}+\mathrm{He}^{+}(1.3-12.5 \mathrm{MeV})$ and $\mathrm{He}^{2+}+\mathrm{He} \rightarrow$ $\mathrm{He}+\mathrm{He}^{2+}(1.6[16]$ and $6.0 \mathrm{MeV})$ collisions. This observation is also reflected in the results of direct mea- 


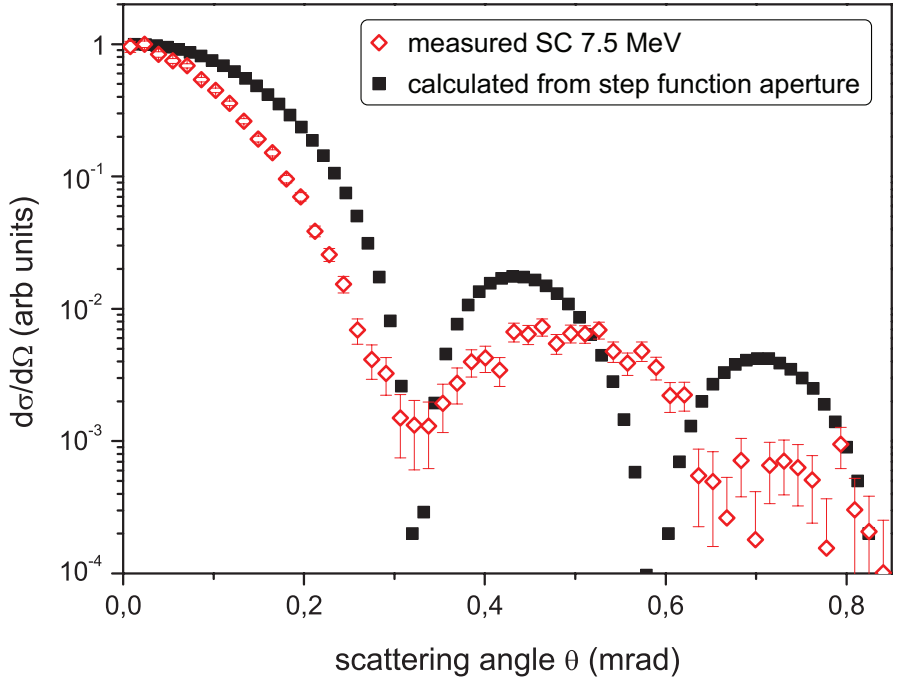

FIG. 7: Measured cross section $d \sigma / d \Omega$ (open diamonds) and cross section resulting from a step-function $P(b)$ (full squares) with radius chosen as to give a minimum in $d \sigma / d \Omega$ at 0.32 mrad.

surements of the variation of the transversal momentum transfer to the projectile, $p_{\perp}$, with the projectile velocity $v_{p}$ and we deduce separate linear $p_{\perp}\left(v_{p}\right)$ scalings for the one- and two-electron transfer processes. These results are markedly different from what would be expected for pure projectile-target nucleus Rutherford scattering. We have shown that it is possible to rationalize these findings by considering diffractions of projectile de Broglie waves in small "apertures" corresponding to electron transfer probabilities, $P(b)$, in narrow regions of impact parameters. The sizes of these regions decrease with increasing projectile velocities. This picture thus readily accounts for the constant widths of the forward scattering peaks and for the appearances of second and third maxima in $d \sigma / d \theta$ in the $\mathrm{H}^{+}+\mathrm{He}$ case. The secondary maxima, and their positions and widths, are however also accounted for through the Thomas p-e-N mechanism and we have shown that there is a quantitative agreement between how the secondary peak is expected to change with $v_{p}[4]$ and our measurement. In this work, we have nevertheless clearly demonstrated that projectile wave diffraction phenomena have to be considered when describing projectile angular scattering of electron capture in fast ion-atom collisions and that the diffraction actually dictate several main features of the corresponding differential cross sections.

Single-aperture diffraction has not been much discussed before in connection with electron capture in fast ion-atom collisions (an exception is [21]) and this is, to our knowledge, the first time that such effects have been demonstrated experimentally. This was possible due to the high resolution and the high luminosity of our recoil ion momentum spectrometer mounted inside the ion stor- age ring CRYRING at the Manne Siegbahn Laboratory, Stockholm University.

\section{ACKNOWLEDGMENTS}

This work is supported by the Knut and Alice Wallenberg Foundation, the Swedish Research Council (VR) and the German Research Council (DFG).

[1] J. Ullrich, R. Moshammer, A. Dorn, R. Dörner, L. P. H. Schmidt, , and H. Schmidt-Böcking, Prog. Phys. 66, 1463 (2003).

[2] J.Ullrich and H. Schmidt-Böcking, Phys. Lett. A 125, 193 (1987).

[3] D. Fischer, K. Støchkel, H. Cederquist, H. Zettergren, P. Reinhed, R. Schuch, A. Källberg, A. Simonsson, and H. T. Schmidt, Phys. Rev. A 73, 052713 (2006).

[4] R. Shakeshaft and L. Spruch, Rev. Mod. Phys. 51, 369 (1979).

[5] J. S. Briggs, P. Greenland, and L. Kocbach, J. Phys. B 15, 3085 (1982).

[6] E. Horsdal-Pedersen, C. L. Cocke, and M. Stockli, Phys. Rev. Lett. 50, 1910 (1983).

[7] L. H. Thomas, Proc. R. Soc. 114, 561 (1927).

[8] R. M. Drisko, Ph.D. Thesis Carnegie Institute of Technology, Unpublished (1955).

[9] D. Fischer, M. Gudmundsson, Z. Berenyi, N. Haag, H. A. B. Johansson, D. Misra, P. Reinhed, A. Källberg, K. Støchkel, H. Cederquist, et al., Phys. Rev. A 81, 012714 (2010).

[10] H. T. Schmidt, H. Cederquist, R. Schuch, L. Bagge, A. Källberg, J. Hilke, K.-G. Rensfelt, V. Mergel, M. Achler, R. Dörner, et al., Hyperfine Interactions 108, 339 (1997).

[11] H. T. Schmidt, A. Fardi, R. Schuch, S. H. Schwartz, H. Zettergren, H. Cederquist, L. Bagge, H. Danared, A. Källberg, J. Jensen, et al., Phys. Rev. Lett. 89, 163201 (2002).

[12] J. Ullrich, R. Moshammer, A. Dorn, R. Dörner, L. P. H. Schmidt, and H. Schmidt-Böcking, Rep. Prog. Phys. 66, 1463 (2003).

[13] K. Støchkel, O. Eidem, H. Cederquist, H. Zettergren, P. Reinhed, R. Schuch, C. L. Cocke, S. B. Levin, V. N. Ostrovsky, A. Källberg, et al., Phys. Rev. A 72, 050703(R) (2005).

[14] M. Lebech, J. C. Houver, and D. Dowek, Rev. Sci. Instrum. 73, 1866 (2002).

[15] K. Abrahamsson, G. Andler, L. Bagge, E. Beebe, P. Carle, H. Danared, S. Egnell, K. Ehrnsten, M. Engström, C. J. Herrlander, et al., Nucl. Instrum. Methods B 79, 269 (1993).

[16] R. Schuch, E. Justiniano, H. Vogt, G. Deco, and N. Gruen, J. Phys. B 24, L133 (1991).

[17] H. Danared, G. Andler, L. Bagge, C. J. Herrlander, J. Hilke, J. Jeansson, A. Källberg, A. Nilsson, A. Paál, K. G. Rensfelt, et al., Phys. Rev. Lett. 72, 3775 (1994).

[18] W. Wiley and I. McLaren, Nucl. Instrum. Methods B 79, 269 (1955). 
[19] H. T. Schmidt, J. Jensen, P. Reinhed, R. Schuch, K. Støchkel, H. Zettergren, H. Cederquist, L. Bagge, H. Danared, A. Källberg, et al., Phys. Rev. A 72, 012713 (2005).

[20] D. Belkić, I. Mančev, and J. Hanssen, Rev. Mod. Phys. 80, 249 (2008).

[21] J. E. Miraglia, R. D. Piacentini, R. D. Rivarola, and A. Salin, J. Phys. B 14, L197 (1981).

[22] H. T. Schmidt, D. Fischer, Z. Berenyi, C. L. Cocke,
M. Gudmundsson, N. Haag, H. A. B. Johansson, A. Källberg, S. B. Levin, P. Reinhed, et al., Phys. Rev. Lett. 101, 083201 (2008).

[23] D. Misra, H. T. Schmidt, M. Gudmundsson, D. Fischer, N. Haag, H. A. B. Johansson, A. Källberg, B. Najjari, P. Reinhed, R. Schuch, et al., Phys. Rev. Lett. 102, 153201 (2009). 